\title{
Laparoscopic salpingectomy for hydrosalpinx in adolescent females-a report of two cases
}

\author{
Jitender Monga $\cdot$ Linga Dwarakanath • \\ Harish Chandran
}

Received: 15 September 2006 / Accepted: 10 May 2007 / Published online: 9 June 2007

(C) Springer-Verlag 2007

\begin{abstract}
Hydrosalpinx is usually a sequel of pelvic inflammatory disease and is rare in adolescence. We report our experience with two cases of hydrosalpinx in adolescent females aged 14 and 15 years. Both the patients had previous histories of abdominal surgery and presented with worsening lower abdominal pain of several months' duration. The first patient had undergone appendicectomy at the age of 3 years, while the second had undergone multiple laparotomies (Beckwith-Wiedemann syndrome, nephrectomy for Wilms' tumour, adhesive intestinal obstruction, etc.) in the past. Magnetic resonance imaging and laparoscopy revealed bilateral hydrosalpinx in the first patient which was treated by laparoscopic bilateral salpingectomy. The second patient underwent a right-sided salpingectomy for a right hydrosalpinx. We discuss the case histories and present a review of the literature.
\end{abstract}

Keywords Hydrosalpinx · Adolescent · Laparoscopy

J. Monga $(\bowtie)$

Heart of England Basic Surgical Training Rotation,

21, Haselwell Drive,

Kings Norton, Birmingham B30 3PQ, UK

e-mail: j_monga@yahoo.com

L. Dwarakanath

Obstetrics and Gynaecology, City Hospital,

Birmingham, UK

H. Chandran

Paedatric Urology, Birmingham Children' Hospital,

Birmingham, UK

\section{Introduction}

Hydrosalpinx is almost always a sequel of previous pelvic infection. It results from the accumulation of secretions from the tubal epithelium when the fimbriated end of the tube becomes occluded. This occlusion can also result from an adjacent appendicular inflammation or previous abdominal surgery. We report on two cases of hydrosalpinx in young girls, both treated by laparoscopic resection.

\section{Case report 1}

A 14-year-old girl was referred to us with lower abdominal pain of 2 months' duration requiring an increasing amount of analgesia. She had regular menstrual cycles and no significant medical history of note. Bowel and micturition habits were normal. Her surgical history included a laparotomy at the age of 3 years for peritonitis secondary to ruptured appendicitis.

Examination revealed tenderness in both iliac fossae. General examination was unremarkable. Transabdominal ultrasound scan showed normal anteverted uterus and complex cystic formations present in both ovaries. The largest was on the right with a $7.6 \mathrm{~cm}$ diameter complex septate cyst or cluster of cysts expanding the right ovary. There was no evidence of internal haemorrhage or solid components. Further small cysts were present in the left adnexa. Laboratory results showed a normal level of white cell count, C-reactive protein and tumour markers. A magnetic resonance imaging (MRI) scan revealed normal follicular changes in both ovaries. Medial to these normalsized ovaries convoluted fluid-filled structures were noted which extended towards the anteverted uterus consistent 
with gross bilateral hydrosalpinx. There was no evidence of free fluid in the pelvis (Figs. 1 and 2).

She underwent a laparoscopic assessment which revealed a normal-sized uterus with obliteration of the pouch of Douglas, filmy omental adhesions to the previous appendicectomy scar and large tortuous retard shaped bilateral hydrosalpinges (Fig. 3). Neither ovary could be visualised. She was discharged $48 \mathrm{~h}$ post-operatively. The findings were discussed with the patient's parents and because of the size of the hydrosalpinges, it was decided to proceed with laparoscopic bilateral salpingectomy. She was readmitted after 4 months for the same. Pneumoperitoneum was created using a Verres needle via Palmer's point with two further ports in both iliac fossae. Per-operatively adhesions were divided using monopolar diathermy. Both hydrosalpinges ruptured during manipulation and were resected. Endoloops (Ethicon, Edinburgh, UK) were placed on the stumps. The post-operative recovery was uneventful and the patient was discharged after 3 days. Histopathological examination confirmed the diagnosis of bilateral hydrosalpinges of no apparent cause.

\section{Case report 2}

A 15-year-old girl presented with recurrent episodes of intermittent right lower abdominal pain of 4 months' duration associated with nausea and vomiting. She was

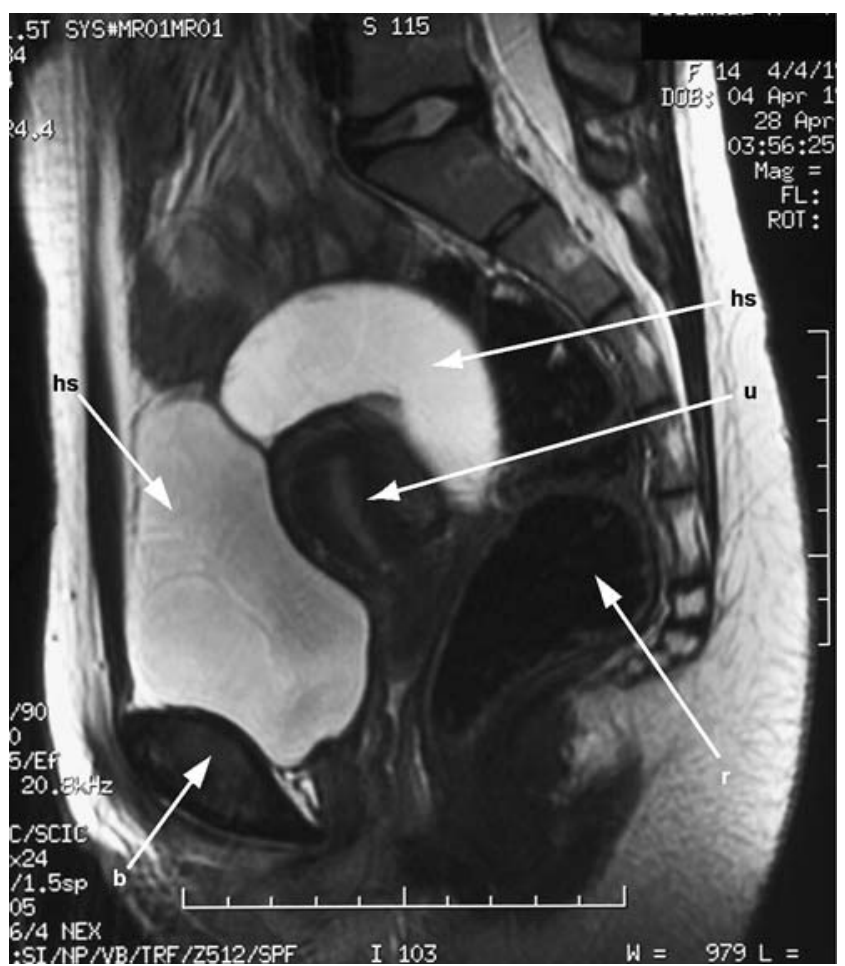

Fig. 1 MRI scan: sagittal section, T2-weighted image demonstrating the hydrosalpinges (patient 1). $u$ uterus, $r$ rectum, $b$ bladder, $h s$ hydrosalpinx

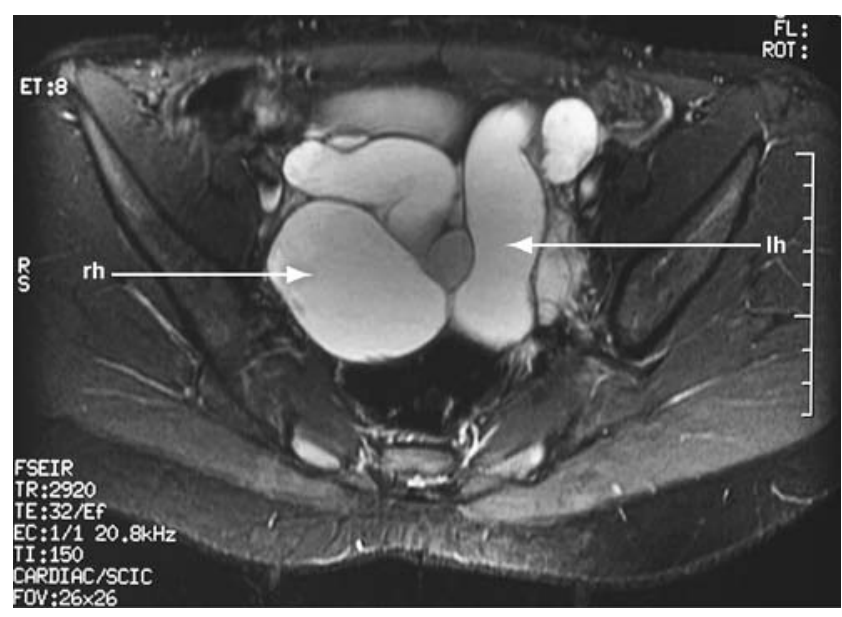

Fig. 2 MRI scan: transverse section, T2-weighted image demonstrating the hydrosalpinges (patient 1). rh right hydrosalpinx, lh left hydrosalpinx

diagnosed with Beckwith-Wiedemann syndrome at 6 weeks of age and had regular ultrasound scans. She was found to have right-sided Wilms' tumour at the age of 8 months and underwent partial nephrectomy followed by vincristine treatment for 12 weeks and a total nephrectomy 2 months later. She underwent laparotomy for intestinal obstruction secondary to bowel adhesions at the age of 13 months. She also had hemihypertrophy of her right lower limb for which an epiphysiodesis was done at the age of 14 years.

Examination revealed deep tenderness in the right iliac fossa with no guarding or rigidity. Laboratory parameters revealed a normal white cell count and normal inflammatory markers. Transabdominal ultrasound scan showed a complex $4.4 \times 4 \times 5.7 \mathrm{~cm}$ thin-walled cystic area to the right of the dome of the bladder and several small cysts in the right ovary. MRI confirmed the same findings of a complex cystic area superior to and to the right of the dome of the bladder. The uterus and left adnexa were normal. There was

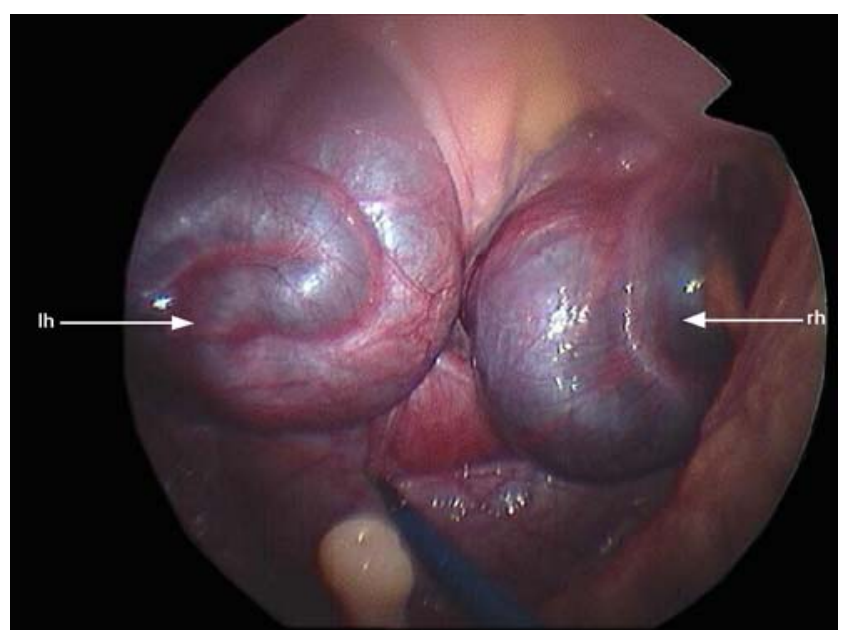

Fig. 3 Laparoscopic view of the pelvis showing the uterus and the hydrosalpinges (patient 1). rh right hydrosalpinx, $l$ left hydrosalpinx 
a tiny trace of free fluid in the pelvis. The serum human chorionic gonadotrophin and alpha fetoprotein were within normal limits. She was taken to theatre for a laparoscopic assessment. A standard laparoscopy was performed using open insertion of the $5 \mathrm{~mm}$ umbilical port and two further ports in the right and the left iliac fossae. Per-operative findings revealed a small amount of pelvic free fluid, large tense hydrosalpinx with several loops of small bowel adherent to it, normal uterus and the left ovary and fallopian tube and a follicular cyst in the region of the right ovary. A decision was taken to proceed with right salpingectomy. Small bowel loops were separated from the hydrosalpinx and it was resected, using a PDS Endoloop to ligate the stump. The resected specimen was retrieved through the umbilical port and sent for histopathological examination. The patient made an uneventful recovery and was discharged on the 3rd post-operative day. The histopathology revealed a hydrosalpinx on the right side. The patient was doing well and was pain free when seen in the clinic post-operatively.

\section{Discussion}

Hydrosalpinx, derived from Greek, literally means "water tube". It is a rare finding in adolescent girls and there are not many cases reported in the literature of a hydrosalpinx in young girls [1]. In the vast majority of patients it is secondary to pelvic inflammatory disease, and the underlying mechanism causing a hydrosalpinx formation is the occlusion of the fimbriated end of the tube adjacent to the ovary which results in distension of the tube with fluid [2]. This occlusion can also occur due to adhesions following an adjacent appendicular inflammation [2]. Our first patient also presented 11 years after an appendicectomy. The other patient had a history of multiple abdominal surgeries, including a laparotomy and adhesiolysis for an intestinal obstruction, and we assume that the cause of hydrosalpinx in this case is the formation of adhesions at the fimbriated end of the fallopian tube secondary to previous surgery.

Transabdominal ultrasound is the initial imaging modality in children or adolescents with acute or subacute pelvic pain. It is relatively inexpensive and provides simple answers to critical questions in the initial phases of work-up of an adolescent girl with pelvic pain. Magnetic resonance imaging should be used for the further characterization of lesions that are indeterminate on the basis of the ultrasound findings [3]. Tubal enlargement can be easily seen on MR images and is characterized by the tortuous infolding of fluid-filled structures on T2 - weighted images [3]. In both of our patients MRI scan was performed which confirmed the diagnosis of hydrosalpinx in the form of convoluted fluidfilled tubular structures.

Laparoscopic surgery is the treatment of choice for adnexal disorders in children and has proved to be an important diagnostic as well as a therapeutic tool. The main advantage is that the laparotomy incision is avoided leading to less post-operative pain and discomfort for the patient, shorter hospitalization time, lower hospital cost, better cosmetic results and quicker resumption of normal activities [2]. Both our patients were discharged 3 days after the surgery without any complications. They were completely pain free when seen in the clinic and were quite happy with the cosmetic result as well.

Hydrosalpinx resection is indicated when there is no functional ipsilateral ovary [1]. In case 1 a bilateral salpingectomy was carried out because of the size of the hydrosalpinges whereas in the second patient there was no functional ovary seen on the same side as the hydrosalpinx on laparoscopic assessment.

We report these two cases because hydrosalpinx is a rare finding in adolescent females. It should be suspected in young girls who present with pelvic pain and have a history of previous abdominal surgery, pelvic infection or adjacent focus of infection such as appendicitis. Awareness of this possibility may allow early pre-operative diagnosis and timely intervention.

\section{References}

1. Sacher P, Meuli M (1997) Laparoscopic removal of hydrosalpinx in a girl: case report. Eur J Pediatr Surg 7:239-240

2. Aboulghar MA, Mansour RT, Serour GI (1998) Controversies in the modern management of hydrosalpinx. Hum Reprod 4(6):882-890

3. Outwater EK, Dunton CJ (1995) Imaging of the ovary and adnexa: clinical issues and applications of MR imaging. Radiology 194 (1):1-18 